\title{
Raisins in human health: A review
}

\author{
Patrizia Restani ${ }^{1}$, Gianfranco Frigerio ${ }^{1}$, Francesca Colombo ${ }^{1}$, Luis Peres de Sousa ${ }^{2}$, Ahmet Altindişli ${ }^{3}$, \\ Raul Francisco Pastor ${ }^{4}$, and Chiara Di Lorenzo ${ }^{1}$ \\ ${ }^{1}$ Dipartimento di Scienze Farmacologiche e Biomolecolari, Università degli Studi di Milano, via Balzaretti 9, 20133 Milan, Italy \\ ${ }^{2}$ Instituto Politecnico de Beja, Rua Pedro Soares, Apartado 6155, 7800-295, Beja, Portugal \\ ${ }^{3}$ Dept. of Agriculture, Ege University, Bornova, Izmir 35100, Turkey \\ ${ }^{4}$ Cuarta Cátedra de Medicina Interna, Hospital de Clínicas “José de San Martín”, Universidad de Buenos Aires, Av Córdoba 2351, \\ Buenos Aires, Argentina
}

\begin{abstract}
In the last years, the scientific research in the field of non-alcoholic grape products has increased significantly. Raisins are often evaluated negatively from the nutritional point of view, mainly for their high sugar content. On the other hand, some in vitro and in vivo studies have suggested that raisins could have healthy effects due to their positive phytochemical profile. The aim of this work was the collection of scientific studies performed in humans to assess critically the health-promoting effects of raisins, as a part of the normal/Mediterranean diet. In most cases, the beneficial effects of raisins have been assessed in intervention studies focused on cardiovascular area, diabetes and oral health, where a decrease in postprandial glycemia and insulinemia both in diabetic and healthy subjects has been observed. The positive effects were generally evident after a short-term consumption of about $70 \mathrm{~g} /$ die of raisins in comparison to a similar quantity of snacks or glucose solution. Surprisingly, some positive findings were shown in oral health. On these bases several findings support the suitability of raisins as a source of healthy compounds for human diet, but limits in the data published till now clearly support the need of new specifically designed trials.
\end{abstract}

\section{Introduction}

Several epidemiological studies have shown that a moderate consumption of wine is associated with the reduction of specific risk factors for chronic pathologies, such as cardiovascular diseases, certain cancers, diabetes and dementia [1].

In particular, a J-shaped relationship has been described between the amount of wine consumed and the level of risk. Nevertheless, the wine market is suffering for a decreasing trend due to the frequent abuse/misuse of alcoholic beverages among both adults and young people. The social problem has been considered with special attention by international organizations, such as WHO [2], and OIV (strategic plan 2015-2019). The "new" social and economical situation has stimulated the scientific research in the field of non-alcoholic grape derivatives (table grapes, grape juices, grape extracts, raisins, etc.), as an alternative source of healthy molecules. Raisins are obtained by drying different cultivar/varieties of Vitis vinifera L.; the Thomson seedless variety (called usually sultana) covers approximately $95 \%$ of the market.

From the nutritional point of view, raisins are often considered negatively due to their high sugar content; as a consequence, their inclusion in the diet of children, obese and diabetic subjects is debated.

On the other hand, raisins have been included in the human diet since ancient times (probably 1400 BC) for their energetic value but also for the presence of specific nutritional compounds. In fact, raisins are rich in sugars (fructose and glucose), minerals (magnesium, iron, potassium, phosphorus, zinc), vitamins (ascorbic acid, pyridoxine, riboflavin and thiamin), dietary fiber, and other active molecules (flavonoids, hydrocinnamic acids, epicatechins, resveratrol, etc.) $[3,4]$.

Some in vitro and in vivo studies have described beneficial effects associated with the consumption of raisins, but the strict causality is often object of discussion. The aim of this review was the collection of scientific papers describing studies performed in humans. When possible, a critical assessment will be elaborated to verify the correlation between the healthy effects considered and the consumption of raisins, as a part of the normal/Mediterranean diet.

\section{Methods}

Two among the most important scientific databases of references and abstracts on life sciences (PubMed/MEDLINE and Embase) were systematically searched (from database inception to June 2016) using the terms "dried grape", "raisins", "sultana" and "Thompson seedless" in combination with "beneficial effects", "health" and refining the results for "human studies".

\section{Results}

The papers reporting data of human studies performed with raisins are quite rare. Most of them describe results in the area of cardiovascular diseases, diabetes and oral health. The protocols used in the most interesting human trials collected and selected for this review are summarised in Table 1. 
Table 1. Study protocols.

\begin{tabular}{|c|c|c|c|}
\hline $\begin{array}{l}\text { Trial } \\
\text { type }\end{array}$ & Subject characteristics & Treatments & Ref \\
\hline $\mathrm{RCOS}$ & 10 healthy sedentary 11 trained, 10 pre-diabetic & $69 \mathrm{~g}$ raisins vs. $50 \mathrm{~g}$ glucose in solution & [5] \\
\hline RCS & $\begin{array}{l}46 \mathrm{M} / \mathrm{F} \text { - adults BMI }=25-35 \text {; fasting glycemia } \\
90-150 \mathrm{mg} / \mathrm{dL}, \mathrm{BDBP}:>80 \mathrm{~mm} \mathrm{Hg} ; \mathrm{BSBP}>120 \mathrm{~mm} \mathrm{Hg}\end{array}$ & $12 \mathrm{w}, 3$ times/d $28 \mathrm{~g}$ raisins vs. equicaloric snack & [6] \\
\hline RCS & 19M, 27F with Type-2 Diabetes & $12 \mathrm{w}, 3$ times/d $28 \mathrm{~g}$ raisins vs. equicaloric snack & [7] \\
\hline $\mathrm{RCOS}$ & $4 \mathrm{M}, 4 \mathrm{~F}$ endurance- trained cyclists age $18-40 \mathrm{y}$ & $1 \mathrm{~g} / \mathrm{kg}$ bw $45^{\prime}$ before exercise raisins vs sports gel & [8] \\
\hline RCS & Healthy $13 \mathrm{M}, 13 \mathrm{~F}$ age $8-11$ y & Water, grape raisins, mix raisins:almond (1:1) & {$[10]$} \\
\hline $\mathrm{RCS}$ & Healthy $17 \mathrm{M}, 17 \mathrm{~F} 50-70 \mathrm{y}$ & $\begin{array}{l}1 \text { cup raisins/day, increasing steps/day, combina- } \\
\text { tion of two }\end{array}$ & [11] \\
\hline $\mathrm{RCOS}$ & Healthy $11 \mathrm{M}, 9 \mathrm{~F}$ age $7-11$ y & $3 \mathrm{~g}$ raisins; $10 \mathrm{~g}$ raisins, bran flakes or raisins bran & [13] \\
\hline RCS & $46 \mathrm{M} / \mathrm{F}$ with pre-hypertension & $12 \mathrm{w}, 3$ times/d raisins vs equicaloric snacks & {$[15]$} \\
\hline RCS & $17 \mathrm{M}$ and 17 postmenopausal $\mathrm{F}$ & $\begin{array}{l}1 \text { cup raisins/day, increasing steps/day, combina- } \\
\text { tion of two }\end{array}$ & [16] \\
\hline $\mathrm{RCOS}$ & $8 \mathrm{M}, 9 \mathrm{~F}, \mathrm{BMI}>26$ age $18-40 \mathrm{y}$ & $14 \mathrm{~d} /$ period $90 \mathrm{~g}$ raisins vs. isocaloric placebo & [17] \\
\hline RCOS & Healthy 15M BMI 20.1-31.7. 18-45 y & $4 \mathrm{w}, 250 \mathrm{~g}$ seedless grape, $50 \mathrm{~g}$ raisins & [18] \\
\hline
\end{tabular}

RCS $=$ Randomized Controlled Study.

RCOS $=$ Randomized Crossover Study

RPCS $=$ Randomized Placebo- Controlled Study.

$\mathrm{BDBP}=$ Baseline Diastolic Blood Pressure.

$\mathrm{BSBP}=$ Baseline Systolic Blood Pressure.

$\mathrm{M}=$ males, $\mathrm{F}=$ female, $\mathrm{y}=$ year, $\mathrm{w}=$ week; $\mathrm{d}=$ day.

\subsection{Raisins, Glycemic Index (GI) and Insulin Index (II)}

Due to their high content of sugar, raisins have been object of debate about their suitability for the diet of prediabetic or diabetic subjects. Kim et al. [5] measured the glycemic index and the insulinemic response after the administration of $69 \mathrm{~g}$ of raisins or a solution containing $50 \mathrm{~g}$ of glucose. The trial included sedentary, trained and pre-diabetic subjects (Table 1). Results are illustrated in Fig. 1: in all groups the area under the curve (AUC) was higher after the administration of glucose solution. The calculated GIs were 49.4, 62.3 and 49.6 for sedentary, trained and pre-diabetic subjects, respectively. Similar results were observed in calculating AUC for serum insulin response (Fig. 2), The calculated Insulin Indexes were 47.1, 51.9 and 54.4 for sedentary, trained and pre-diabetic subjects, respectively. The authors concluded that GI of raisins should be considered from low to moderate so that, also considering the general nutritional properties, raisins should be considered a healthy snack food.

In another study, Anderson et al. [6] showed that $28 \mathrm{~g}$ of raisins administered 3 times/day for 12 weeks decreased significantly postprandial glycemia by $13.1 \mathrm{mg} / \mathrm{dL}$, while no change was observed when common non-fruit snack was used (see Table 1 for protocol). Moreover, only raisins determined a significant reduction of glycated haemoglobin $(\mathrm{gHb})$ level by $0.12 \%$.

The same group of researchers performed a trial with the same protocol in patients suffering from type2 diabetes mellitus [7]. A similar statistical significant reduction of post-prandial glucose level was observed; the decrease of $\mathrm{gHb}$ levels, even though present, was not of statistical significance.

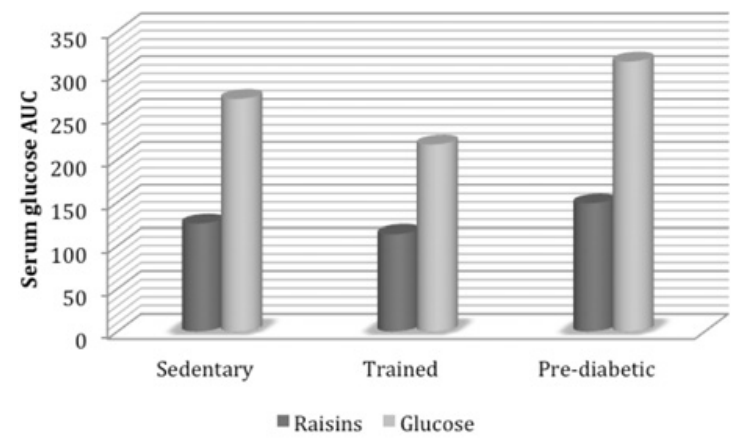

Figure 1. Serum glucose AUC (mmol/L min) after administration of raisins or glucose solution (modified from Ref. 5).

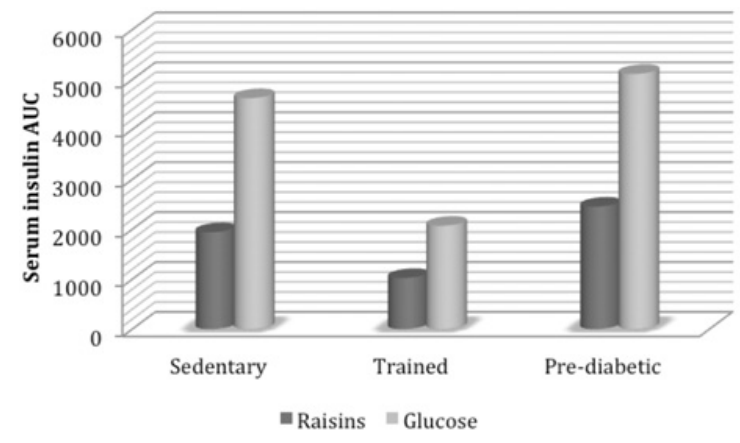

Figure 2. Serum insulin AUC ( $\mu \mathrm{UI} / \mathrm{L}$ min) after administration of raisins or glucose solution (modified from Ref. 5).

\subsection{Raisins in metabolism and sport performance}

Even though object of debate, some researchers showed that the consumption of a lower Glycemic Index (GI) 


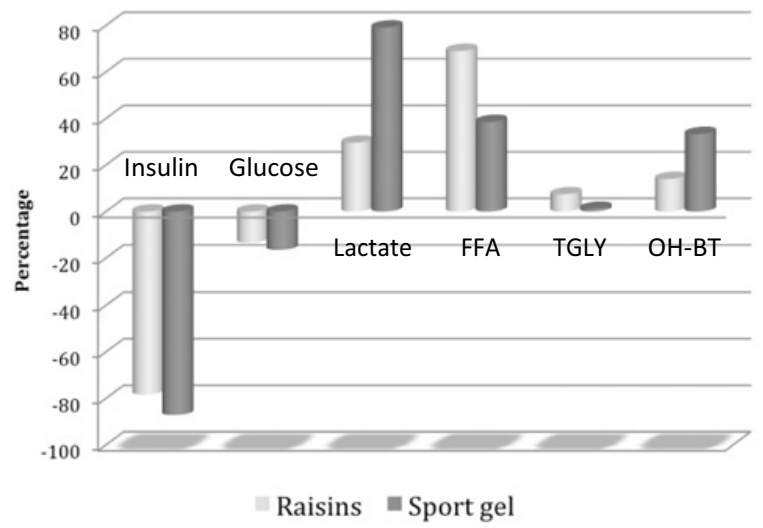

Figure 3. Changes in percentage between pre- and post-exercise on serum parameters (modified from Ref. 8 ). FFA $=$ Free Fatty Acids; TGLY $=$ Triglycerides; $\mathrm{OH}-\mathrm{BT}=\beta$-Hydroxy-Butirate.

food before sport activity was more effective than high GI food in improving performances. On these bases, Kern et al. [8] assessed the efficacy of a moderate GI food (raisins; GI=88) and a high GI food (a commercial sport gel; $\mathrm{GI}=117$ ) in modulating the metabolism and performance in endurance-trained cyclists. Four males and four females were included in the study; they received $1 \mathrm{~g} / \mathrm{kg}$ body weight of bioavailable sugars with raisins or with commercial sport gel (Table 1). After $45^{\prime}$ minutes in seated position, athletes started cycling for $45^{\prime}$. Each subject received both foods, in two trials separated by at least 7 days.

Some serum parameters were measured and their changes between pre- and post-exercise are illustrated in Fig. 8.

Although the foods studied determined different glycemic responses during rest $(\mathrm{GI}=66$ and 88 for raisins and sports gel, respectively), few differences were observed in metabolism when raisins and sports gels were consumed before the cycling trial. The results of this study did not support the hypothesis at the origin of the study stating that the consumption of a lower GI food before the training could enhance performances when compared to high GI foods. In fact, minimal differences were observed in performance during the two trials. As a consequence the authors conclude: "Raisins appear to be a cost-effective source of carbohydrate for pre-exercise feeding in comparison to sports gel for short-term exercise bouts".

Similar results were published by Apfel and coworkers [9], who assessed the physical performance of 11 male athletes receiving randomly raisins, commercial sport chews and water. Interventions were administered during the physical activity based on 80-minute treadmill run, followed by $5 \mathrm{~km}$ timed trial. Compared to water, performances were similar when athletes received raisins or sport chews. On this bases and considering the nutritional quality, authors considered raisins a better energetic snack if compared to sport chew.

\subsection{Raisins and food intake}

Patel et al. in their study [10] assessed the effect of including dried fruit into the diet of children in term of energy and Food Intake (FI). A within subject, randomized, repeated-measures design was used to measure FI after consumption of an ad libitum or a fixed- calorie snack containing: 1) grape, 2) raisins, 3) 1:1 mix of raisins and almonds, or 4) ad libitum water as a control (Table 1). The overall conclusion of the complex study indicates that the consumption of a pre-meal snack containing raisins (but not those with grape or the mix raisins/almonds) reduces meal-time energy intake. This means that raisins could be consumed as an alternative source of valuable nutrients (fibre, antioxidants, etc.) for children.

Another study by Puglisi et al. [11] assessed the effects of: 1) a consumption of 1 cup raisins/day, 2) an increased number of steps walked, and 3) a combination of two interventions in modulating hunger and satiety in a group of adult subjects (Table 1). The parameters measured were: plasma apoliproprotein concentrations, cholesterol ester transfer protein activity, LDL receptor messenger RNA abundance, ghrelin and leptin concentrations. All interventions (raisins, walking or combination) modulated lipoprotein metabolism, which could reduce the risk for cardiovascular diseases. Raisins, but not walking, increased plasma level of leptin, which could modulate satiety and decrease caloric intake. The parallel increase in plasma ghrelin could be associated with a decreased intake of food due to the greater level of leptin. Moreover, a decreased expression of LDL receptor in mononuclear cells could be responsible for a reduction of LDLcholesterol. Walking, but not raisins, reduced apo C-III, which could contribute in reducing plasma triglycerides. The conclusion of the authors was: "Easily implemented lifestyle changes, such as increasing raisins consumption or walking additional steps each day may serve as effective interventions to promote weight control and lower CVD risk".

\subsection{Raisins and dental plaque}

Dental decay is a significant source of pain in children and young people. Raisins have been usually considered a promoting food for caries, due to their sticky characteristics. Rose et al. used an in vitro method to monitor the effect of raisins on tooth enamel demineralisation [12]. Slices of caries-free human molars were inoculated with Streptococcus mutants for 2 hours to allow bacterial adhesion. Then, slices were exposed to saliva-like solution without (control) or with $10 \%$ raisin juice 15 minutes/day for 7 days. Authors concluded that raisin juice might promote bacteria activity, which is responsible for teeth demineralization. Important to underline that differences with control group were not statistically significant.

Utreja et al. studied the effect of raisin-containing cereals on plaque acidogenicity, a factor involved in the dental caries progression [13]. Twenty healthy children (7-11 years; 11 males and 9 females) were enrolled in a randomized, controlled, crossover study (Table 1). Subjects received 4 test foods once a week: raisins as such (3 g); commercial bran flakes (10 g); commercial and experimental raisin bran cereal mixtures (10 g containing $3 \mathrm{~g}$ of raisins). Sucrose and sorbitol solutions (10\%) were used as a positive and a negative control, respectively. In vivo plaque $\mathrm{pH}$ was measured at baseline and 2, 5, 10, 


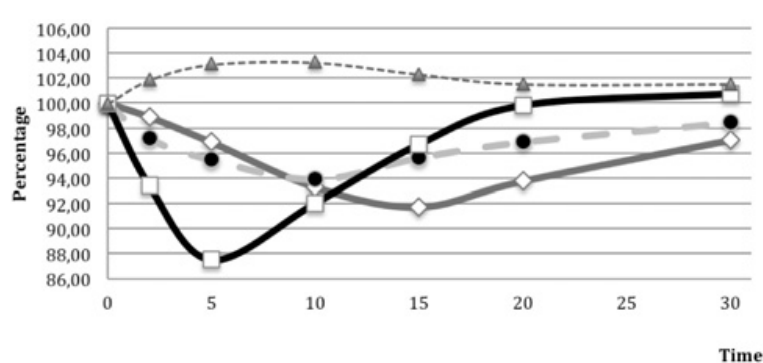

Figure 4. Changes in dental plaque $\mathrm{pH}$ in children after the consumption of bran $(\diamond)$, raisins $(\bullet)$, or the rinsing with sucrose as a positive control $(\square)$ and sorbitol as a negative control ( $\mathbf{\Delta})$. Modified from Ref. 13.

15, 20 and 30 minutes after food consumption or control solution application.

Figure 4 illustrates the pattern of plaque $\mathrm{pH}$ during a period of 30 minutes from the intake of food or the rinsing with control solutions.

Positive (sucrose) and negative (sorbitol) controls modified the plaque $\mathrm{pH}$ profile in opposite way; sucrose determined a fast reduction of $\mathrm{pH}$ with a negative peak after 5 minutes $(-12.5 \%)$, while sorbitol did not interfere significantly with the same parameter during the whole period tested. Bran and raisins modified the plaque $\mathrm{pH}$, reducing the value by only 8.3 and $6.1 \%$, respectively. When the experimental mixture of bran and raisins was used, the effect on plaque $\mathrm{pH}$ was positively affected, allowing concluding that the consumption of bran, raisins or mixtures of them (without added sugar) does not represent a risk factor for dental decay.

Other authors [14] supported the positive role of raisins on oral health showing, by an in vitro approach, that raisins are reach in compounds with antibacterial activity versus Streptococcus mutants and Porphyromonas gengivalis, which are involved in dental decay. Among molecules capable of inhibiting oral pathogens there were: oleanolic acid, oleanolic aldehyde, linoleic acid, linolenic acid, betulin, betulinic acid, 5-hydroxymethyl-2- furfural, rutin, $\beta$-sitosterol, and $\beta$-sitosterol glucoside.

\subsection{Raisins and cardiovascular diseases}

\subsubsection{Blood pressure}

Cardiovascular diseases are associated with several risk factors and some of them were the objects of papers reporting the intake of raisins. In the same trial described above (see Table 1), Anderson et al. monitored the effect of the consumption of raisins on blood pressure compared to an isocaloric snack [6]. Raisins decreased the systolic blood pressure (SBP) by 6 to $10.2 \mathrm{~mm} \mathrm{Hg}$ (statistically significant) and diastolic blood pressure (DBP) by 2.6 to $5 \mathrm{mmHg}$. Figures 5 and 6 illustrate changes in the values of SBP and DBP, respectively.

Comparing two groups receiving raisins or an isocaloric snack, Bays et al. [7,15] showed that raisins significantly reduced the systolic blood pressure in a group of patients with type- 2 diabetes mellitus $(-7.5 \%)$. On the other hand, no significant improvement of diastolic blood pressure was observed.

Similar conclusions were reached by Puglisi and coworkers [16] who enrolled 34 men and postmenopausal

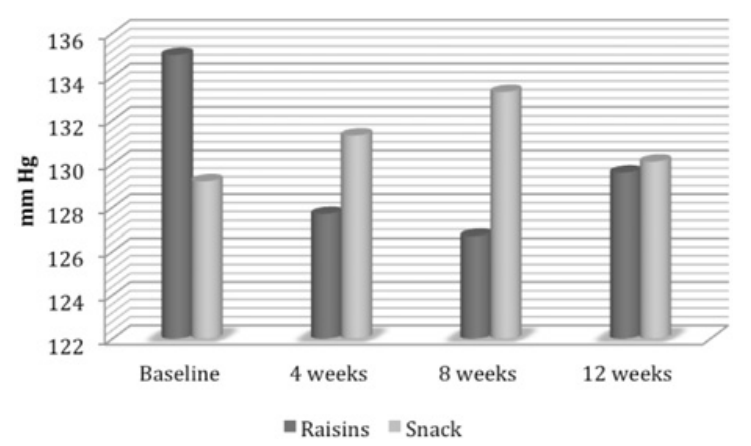

Figure 5. Mean values of the systolic blood pressure measured before and after the consumption for 4-12 weeks of raisins or an isocaloric snack (modified from Ref. 6).

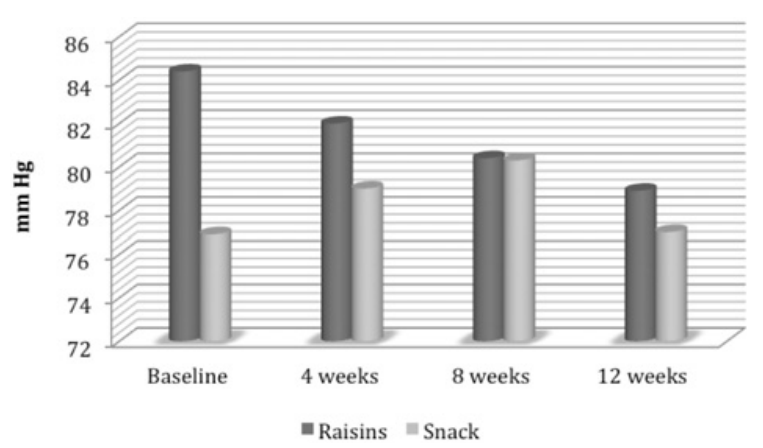

Figure 6. Mean values of the diastolic blood pressure measured before and after the consumption for 4-12 weeks of raisins or an isocaloric snack (modified from Ref. 6).

women in a trial where raisins ( 1 cup/day) was compared to physical activity (increased number of steps walked) or a combination of two (see Table 1). In fact, there was a significant reduction by $2.2 \%$ of systolic pressure in all intervention groups, while diastolic pressure was unchanged.

\subsubsection{Plasma lipids}

Total cholesterol, LDL-cholesterol and triglycerides are well-known risk factors for cardiovascular diseases. Puglisi et al in the trial cited above [16] observed a significant reduction of total cholesterol and LDLcholesterol in all groups after 6 weeks from the beginning of the study. As shown in Fig. 7, the efficacy of intervention was: step walking $>$ raisins $>$ combination of two. No significant change was observed for HDL- cholesterol; only group of walking showed a reduction of serum triglyceride level.

Barnes and co-workers [17] measured the level of circulating oxidized LDLs, which is a risk factor for Coronary Artery Disease (CAD). Participants $(n=32)$ were distributed randomly in three intervention groups, receiving daily 56, 99 and $157 \mathrm{~g}$ of raisins for 4 weeks. Blood samples were drawn at baseline, and after 2 and 4 weeks of raisin consumption. The intake of $99 \mathrm{~g} / \mathrm{day}$ of raisins decreased significantly the concentration of circulating oxidized LDL after 4 weeks of intervention. Oxidized LDL level and plasma antioxidant capacity (FRAP assay) were significantly and positively modified after 2 weeks in the group receiving $157 \mathrm{~g}$ /day of raisins. 


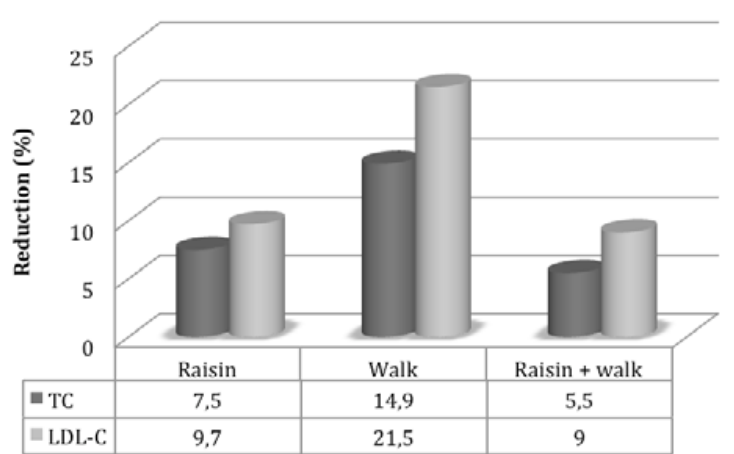

Figure 7. Mean reduction of Total cholesterol (TC) and LDLCholesterol (LDL-C) after 6 weeks from the beginning of intervention (modified from Ref. 16).

\section{Discussion}

Summarizing the data described in this review, it is possible to identify some positive effects associated with the consumption of raisins:

1. a positive modulation of postprandial glycemia and insulinemia, observed also in subjects with type-2 diabetes mellitus;

2. a modulation of hunger and satiety, with a possible contribution in the body weight control;

3. a possible role in reducing dental decay, due to its limited contribution to the plaque acidogenicity and to its content in active molecules capable of inhibiting oral pathogens;

4. a reduction of systolic pressure, total cholesterol and LDL-cholesterol, important risk factors for cardiovascular diseases.

Some biochemical mechanisms have been proposed for justifying the healthy effects of raisins and the most important scientific contributions indicate that antioxidant and antinflammatory activities could be responsible for the positive modulation observed in human studies.

Puglisi and co-workers [16] suggested an involvement of the antioxidant properties by raisin polyphenols and the positive modulation of antiinflammatory markers, such as cytokines and TNF- $\alpha$. Following the protocol described before (see also Table 1), Authors showed a significant modification of some parameters, such as the plasma concentration of SICAM-1 in all intervention trials (raisins, walking or combination of two). The reduction of sICAM1 could modulate positively the adhesion of monocytes to the vascular endothelium, preventing the progression of atherosclerosis. TNF- $\alpha$, which is an important cell signalling protein involved in inflammation, was lowered significantly from 3.5 to $2.1 \mathrm{ng} / \mathrm{L}$ in the group receiving raisins.

These mechanisms are partially confirmed by other authors, who performed in vivo, in vitro or ex vivo studies. Rankin et al. [18] enrolled 17 subjects overweight for a trial, where the effects of raisins (90 g) were compared to those of an isocaloric placebo (Table 1). The study was performed as a randomized cross-over protocol based on 14-days periods in which subjects followed a diet poor in flavonoids, and received raisins, or placebo, associated with a daily diet distributed in 4 high-fat $(53 \%)$ meals. Several biological activities and biomarkers were measured before and after the intervention/placebo

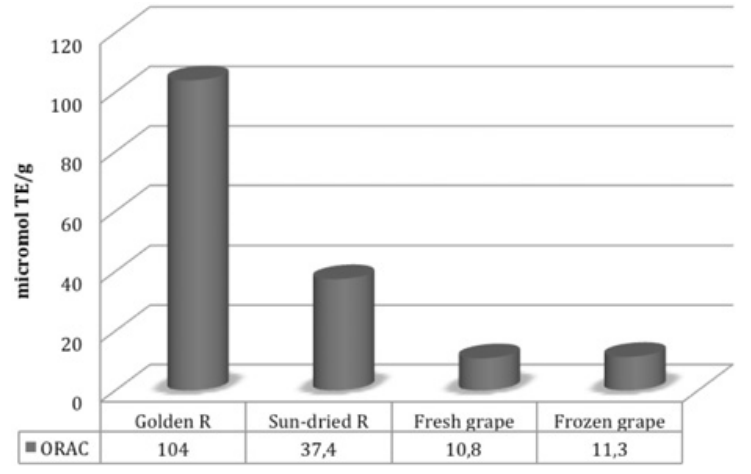

Figure 8. Mean ORAC values measured in raisin (R) and grape samples (modified from Ref. 19).

period: urinary 8-isoprostaglandin- F-2 $\alpha$ as a marker of oxidative stress; serum oxygen radical absorbance capacity (ORAC); serum C-reactive protein, interleukin-6 and Tumour Necrosis Factor- $\alpha$ (TNF- $\alpha$ ), as inflammatory markers; serum soluble intercellular adhesion molecule-1 (sICAM-1) and soluble vascular cell adhesion molecule1 as endothelial markers; free fatty acids, triacylglycerol, glucose and insulin response, as metabolic markers. This study showed that raisins were capable of increasing moderately the fasted serum total antioxidant capacity, without any significant alteration of oxidative stress or inflammatory markers. No significant difference versus control was observed on postprandial response to the highfat meals. On the other hands, the controlled feeding modulated positively a number of biomarkers of oxidative stress and inflammation regardless of treatment.

Other studies based on ex vivo or in vitro approaches produced new insights on this complex topic. Parker and co-workers [19] compared the antioxidant capacity and phenolic profile of some Thompson grape-deriving samples: seedless grape, sun-dried raisins and goldenraisins (see Table 1 for subject characteristics). After 1 week free from any supplement or food containing phenolic compounds, participants (5M/group) received randomly for 4 weeks $250 \mathrm{~g}$ fresh Thompson seedless grapes, or $50 \mathrm{~g}$ of sun-dried raisins or $50 \mathrm{~g}$ of golden raisins. Blood samples were collected each week at baseline, 1 and $2 \mathrm{hr}$ after the consumption of relative "treatment". After three weeks of washout period, participants were moved to a different treatment. The following parameters were measured and compared: serum antioxidant capacity (Oxygen Radical Absorbance Assay, ORAC); ex vivo copper-induced serum lipoprotein oxidation (spectrophotometric assay); total plasma phenolic concentration (Folin-Ciocalteu method); C- reactive protein assay (ELISA method).

Samples were tested for their antioxidant capacity by ORAC analysis and results are illustrated in Fig. 8. Golden raisins showed a very high ORAC value compared to sun-dried raisins; this could be due to the process applied to golden raisins, which determines the protection of antioxidant polyphenols: hot water and SO2 to inactivate polyphenol oxidase and to inhibit nonenzymatic browning. The serum ORAC showed a positive statistically significant increase for grapes after 2 weeks and golden raisins after 3 weeks. Serum oxidation changelag time, a measure of slow/rapid onset of oxidation, 
was positively increased after 4 weeks of golden raisin intake. No significantly change was observed in: plasma total phenolic and C-reactive protein levels. The authors concluded that the daily consumption of grape or raisins, associated with a diet high in carbohydrate, might not be efficient enough in overcoming the oxidative stress observed in the postprandial period. On the other hands, their inclusion in the diet could have healthy antioxidant effects over time. One of the most important results of this paper is the identification of a very critical point, which could also represent the reason of conflicting results obtained in human trials: the lack of information on the variety and composition of samples included in the studies.

As showed by Di Lorenzo et al. [3] in their in vitro study, the biological activity of raisins can change significantly according to their botanical (presence or not of seeds) and chemical (polyphenol pattern) characteristics.

In that paper, the anti-inflammatory activity of 5 raisin samples (characterized for variety and chemical fingerprints) was compared at gastric level, focusing on interleuchin-8 (IL-8) and Tumour Necrosis Factor-Alfa (TNF- $\alpha$ ) pathways. Samples included: seedless Early Gold sultana-like raisin from Portugal; sultana raisin with seeds from Turkey; three commercial seedless samples. Considering the anti-inflammatory properties, the most active raisin sample was the Turkish raisin, which significantly inhibited TNF- $\alpha$-induced IL- 8 release thanks to the impairment of corresponding promoter activity in human gastric epithelial cells. Although the main effect was associated to the presence of seeds, the fruit showed significant activity as well. The conclusion of the authors underlines the necessity to identify suitable raisin varieties to optimize the protection against gastric inflammatory disorders.

\section{Conclusions}

Overall, the conclusions deriving from data collected in this review are in agreement with those by Anderson et Waters [20]: the use of raisins could contribute in reducing some risk factors for developing oral decay, diabetes and cardiovascular diseases. In other words, the inclusion of raisins in a well balanced diet can contribute in promoting human health.

\section{References}

[1] M. Boban, C.Stockley, P-L. Teissedre, P. Restani, U. Fradera, C. Stein-Hammer, J-C. Ruf. Food Funct. 7, 2937 (2016)
[2] WHO. Global strategy to reduce the harmful use of alcohol. 2010. http://www.who.int/ substance_abuse/alcstratenglishfinal.pdf

[3] C. Di Lorenzo, E. Sangiovanni, M. Fimagalli, E. Colombo, G. Frigerio, F. Colombo, L. Peres de Sousa, A. Altindisli, P. Restani, M. Dell'Agli. Int. J. Mol. Sci. 17, 1156, 2016

[4] USDA Nutrient Database for Standard Reference, May 2016. Available at: https://ndb. nal.usda.gov

[5] Y. Kim, S.R. Hertzel, H.K. Byrne, C.O. Mattern. Nutrition Res. 28, 304, 2008

[6] J.W. Anderson, K.M. Weiter, A.L. Christian, M.B. Ritchey, H.E. Bays. Postgraduated Medicine 37, 2014

[7] H. Bays, K. Weiter, J. Anderson. Phys. Sportmed. 43, 37,2015

[8] M. Kern, C.J. Heslin, R.S. Rezende. J Strength Conditioning Res. 21, 1204, 2007

[9] K.E. Apfel, J. Painter, A. Waters, B. Too, S. Cicia, K. Hockett, E. Applegate, B. Davis, G. Casazza. J. Academy of Nutrition and Dietetics 113 (suppl.), A21, 2013

[10] B.P. Patel, B. Luhovyy, R. Mollard, J.E. Painter, G. H. Anderson. Appl. Physiol. Nutr. Metab. 38, 382, 2013

[11] M.J. Puglisi, G. Mutungi, P.J. Brun, M.M. McGrane, C. Labonte, J.S. Volek, M.L. Fernandez. Metabolism Clin. Exp. 58, 120, 2009

[12] J.L. Rose, K.M. Hoffmann, B.D. Schmuck, S.H. Dickens, C.M. Carey. Abstract available at: http:// www . calraisins .org/wp-content/uploads / 2014/01/34-Rose-2008-Raisin-Effects-onin-vitro-Demineralization-of-HumanTeeth.pdf

[13] A. Utreja, P. Lingstrom, C.A. Evans, L.B. Salzmann, C.D. Wu. Pediatric dentistry 31, 498, 2009

[14] C.D. Hu. J Nutrition 139, 1818S, 2009

[15] H.E. Bays, K. Schmitz, A. Christian, M. Ritchey, J. Anderson. JACC 59 (13 s1), E1721, 2012

[16] M.J. Puglisi, U. Vaishnav, S. Shrestha, M. TorresGonzales, R.J. Wood, J.S. Volek, M.L. Fernandez. Lipids in Health and Disease 7, 14, 2008

[17] J.L. Barnes, D.D. Schramm, C. Keen, J.E. Painter, A.R. Waters. J Am Diet Assoc 111:A46, 2011. Available at: http://www.andjrnl.org/ article/S0002-8223(11)00872-8/pdf

[18] J.W. Rankin, M.C. Andreae, C-Y. Oliver Chen, S.F. O'Keefe. Diabetes, Obesity and Metabolism 10, 1086, 2008

[19] T.L. Parker, X-H. Wang, J. Pazmino, N.J. Engeseth. J. Agric. Food Chem. 55, 8472, 2007

[20] J.W. Anderson, A.R. Waters.Journal of Food Science 78 (S1), A11-A17, 2013 